\title{
BMJ Open Association between functional social support and cognitive function in middle-aged and older adults: a protocol for a systematic review
}

\author{
Emily C Rutter, ${ }^{1}$ Suzanne L Tyas, ${ }^{1}$ Colleen J Maxwell (D) , , ${ }^{1,2}$ Jane Law, ${ }^{1,3}$ \\ Megan E O'Connell, ${ }^{4}$ Candace A Konnert, ${ }^{5}$ Mark Oremus (D) ${ }^{1}$
}

To cite: Rutter EC, Tyas SL, Maxwell CJ, et al. Association between functional social support and cognitive function in middle-aged and older adults: a protocol for a systematic review. BMJ Open 2020;10:e037301. doi:10.1136/ bmjopen-2020-037301

- Prepublication history and additional material for this paper are available online. To view these files, please visit the journal online (http://dx.doi org/10.1136/bmjopen-2020037301).

Received 27 January 2020 Revised 14 February 2020 Accepted 20 March 2020

Check for updates

(C) Author(s) (or their employer(s)) 2020. Re-use permitted under CC BY-NC. No commercial re-use. See rights and permissions. Published by BMJ.

For numbered affiliations see end of article.

Correspondence to

Dr Mark Oremus;

moremus@uwaterloo.ca

\section{ABSTRACT}

Introduction Maintenance of cognitive function into old age is important for ageing populations. Researchers seek to identify modifiable risk and protective factors for cognitive function. One such modifiable factor is functional social support, that is, one's perception of whether their social network can provide resources such as material help, companionship, information and emotional contact, if needed. While the literature generally reports positive associations between functional social support and cognitive function, results vary according to study methods such as the tool used to measure functional social support or the specific cognitive domain under investigation. Our review will summarise the association between functional social support and cognitive function in middle-aged and older-aged adults who reside in any setting (eg, community dwelling, long-term care facilities). We will also identify sources of discrepant findings between studies.

Methods and analysis This protocol was reported according to the Preferred Reporting Items for Systematic Reviews and Meta-Analysis Protocols guideline. PubMed, PsycINF0, Sociological Abstracts, Cumulative Index of Nursing and Allied Health Literature (CINAHL) and Scopus will be searched from inception to the present using a search strategy developed with a medical librarian's help. We will supplement the database searches with a grey literature search. English-language or Frenchlanguage studies with a comparison group will be subject to inclusion, regardless of the measures used to assess functional social support or cognitive function. We will assess risk of bias with the Cochrane Risk of Bias ToolVersion 2 or the Newcastle-0ttawa Scale, narratively synthesise the extracted data and conduct a meta-analysis of studies with similar characteristics (eg, sample age and sex, cognitive function outcomes). Two independent raters will screen articles and assess risk of bias.

Ethics and dissemination This review is timely given the push toward early diagnosis and treatment of dementia/ major neurocognitive disorder and other types of cognitive impairment. This protocol does not require a formal ethics review. We will publish our findings in a peer-reviewed journal.
Strengths and limitations of this study

- Our systematic review and meta-analysis will be the first review to focus exclusively on the association between functional social support (overall and subtypes) and cognitive domains (eg, memory, executive function) or diagnostic states (eg, mild cognitive impairment/mild neurocognitive disorder, dementia/ major neurocognitive disorder).

- This protocol adheres to the Preferred Reporting Items for Systematic Review and Meta-Analysis Protocols guideline.

- We will search the grey literature in addition to PubMed, PsycINF0, Sociological Abstracts, CINAHL and Scopus, and include articles published in English or French.

- Two independent reviewers will screen retrieved citations, extract data and assess risk of bias.

- Meta-analysis could be challenging because of potential heterogeneity in methods for measuring social support and cognition.

\section{INTRODUCTION}

Preserving cognitive health and helping people maintain functional independence has become an increasing priority for individuals and care providers as populations age. Lower scores on measures of cognitive function are associated with increased frailty ${ }^{1}$ and limitations to activities of daily living. ${ }^{2}$ Uncovering potentially modifiable risk or protective factors for cognitive decline is key to preventing or managing clinical outcomes such as dementia/major neurocognitive disorder or mild cognitive impairment/mild neurocognitive disorder, and for managing normal, age-related declines incognition.

Several reviews have investigated the association between one such modifiable factor, social support and the preservation of cognitive function. ${ }^{3-7}$ However, the concept of social support is multifaceted and definitions 
have varied considerably across studies, making conclusions from this literature difficult.

Broadly, measures of social support assess either structural or functional features of support, which are overlapping but distinct processes. ${ }^{8}$ Structural social support refers to the presence and objective quantity of potential support sources one may access, ${ }^{8}$ with common measures of this construct including marital status, size of social network (eg, number of relatives or friends) and amount of social activity or community involvement.

Functional social support, in contrast, assesses whether the perceived availability of social resources such as the people in one's social network fulfil certain functions in times of need (eg, provide emotional support when sad, provide transportation to medical appointments). Functional social support has been hypothesised to positively impact physical and psychological health by buffering the effects of stressful events and by increasing positive psychological factors such as self-worth; however, the exact pathways are not known. ${ }^{9}$ Several distinct subtypes of functional support have been identified, including emotional, informational, tangible and affection support, as well as positive social interactions. ${ }^{8}$

The association between structural support and cognitive function has been investigated more frequently ${ }^{10-19}$ than that of functional support, but there is growing evidence for the importance of functional support ${ }^{20-27}$ which may more accurately assess the level and perceived value of support available to an individual.

Previous reviews have demonstrated a relationship between structural social support and cognitive function ${ }^{37}$ and dementia/major neurocognitive disorder. ${ }^{4}$ However, fewer reviews have investigated the impact of functional social support on cognition ${ }^{35}$ and, to our knowledge, only the review by Kelly et al on the impact of social activities, social networks, social support and social relationships on cognitive function in healthy older adults has included discussion of functional social support subtypes. ${ }^{5}$ They found variation in the association between cognitive function and subtypes of functional social support, with emotional support demonstrating stronger associations than instrumental or informational support. However, the number of included articles on functional social support was limited $(n=9)$, prohibiting the authors from thoroughly assessing the associations between each functional subtype and cognitive function (eg, only one study included the subtype of positive social interactions).

In the 3 years since Kelly et als literature search ended (January 2017), ${ }^{5}$ primary studies have reported inverse associations between high levels of certain subtypes of functional social support and cognitive function. ${ }^{27}{ }^{28} \mathrm{In}$ contrast, other studies have found positive associations between functional support subtypes and cognitive function. ${ }^{2324}$ Therefore, the time is ripe for an updated systematic review and meta-analysis to better understand the association of functional social support and its subtypes with cognitive function. This is especially timely in light of the push towards early diagnosis and management of dementia/major neurocognitive disorder, an approach that is predicated on the existence of adequate functional social supports. ${ }^{29}$

This review will build on the foundational work of previous reviews in three ways. First, we will focus specifically on the association between (1) functional social support and its subtypes and (2) cognitive function, thereby allowing us to assess one aspect of social relationships in depth while concomitantly investigating a variety of cognitive outcomes in different populations. Second, by including any study design with a comparison group, we will address the exclusion of articles from previous reviews, which were limited to longitudinal studies. ${ }^{3-5}$ Third, we will employ broad search terms and review the grey literature to potentially generate a large number of included studies and identify results that previous reviews could not.

\section{Objectives}

The proposed review and meta-analysis will investigate whether functional social support is associated with cognitive domains (eg, memory, executive function) and states (eg, mild or major neurocognitive disorder), and whether this association differs across functional social support subtypes. Where possible, we will conduct subgroup analyses by men and women, community versus institutional residence and length of follow-up.

\section{METHODS AND ANALYSIS}

\section{Protocol and registration}

We followed Preferred Reporting Items for Systematic Review and Meta-Analysis Protocols (PRISMA-P) guidelines to report this protocol and included the checklist as online supplementary file 1 . The completed review will be reported in accordance with the PRISMA guidelines. ${ }^{30-32}$

\section{Study eligibility criteria \\ Participants}

All studies with human participants in mid-to late-life (age 40 years or over) will be considered for inclusion. This wide age range will maximise our ability to observe differing associations across the age spectrum and over time. We will also include studies regardless of participants' residential setting (eg, community dwelling, longterm care facilities).

\section{Study design}

We will include all primary studies containing a comparison group (eg, cross-sectional, cohort and case-control study designs, and randomised controlled trials). We will exclude abstracts, editorials, narrative reviews, systematic reviews/meta-analyses, protocols and letters to the editor.

\section{Outcome}

We will include articles reporting cognitive function globally, by domain (eg, memory, executive function), by test (eg, Rey Auditory Verbal Learning Test, ${ }^{33}$ Animal Fluency 
$\mathrm{Tes}^{34}$ ) or by diagnosis of cognitive states (eg, mild or major neurocognitive disorder).

\section{Exposure}

All measures of functional social support which assess perceived availability of social support will be included in the review. These measures may include validated tools such as the Medical Outcomes Study-Social Support Survey. ${ }^{35}$ We will also include articles whose authors purport to study functional social support using other possible measures matching the definition of the construct. We will investigate functional social support as an overall concept and by subtype. Gradients of functional social support will be used as comparators (eg, high vs low support, medium vs low support, change in continuous social support scale scores).

\section{Search strategy}

The following electronic databases will be searched from inception (the earliest date for which citations are indexed in the database in question) to January 2020, with monthly updates undertaken prior to final analysis: PubMed, PsycINFO, Sociological Abstracts, CINAHL and Scopus. Grey literature databases (eg, Google Scholar) will also be searched to retrieve unpublished studies, government or stakeholder reports, and conference papers. Results will be limited to English-language or French-language publications. No limitations will be set on geographical location of studies or publication dates. A medical librarian assisted in the development of the syntax for the PubMed search (online supplementary file 2 ). The syntax will be adjusted accordingly for use with the other databases.

\section{Study selection}

The citations retrieved in the literature search will be screened independently by two raters, who will evaluate each citation against questions based on the eligibility criteria: article includes a comparison group, cognitive function outcome, functional social support exposure and adults aged 40 years or over. Initial screening will be undertaken at the title and abstract level, and articles generating a mix of 'yes' or 'uncertain' responses to the eligibility questions will be promoted to full-text screening. Articles will be included in the review if they garner yes responses to each eligibility question at the full-text screening level. Raters will resolve disagreements by consensus, and a third rater will arbitrate in the event consensus is unreachable.

\section{Data extraction}

Two raters will independently extract data from the final set of included articles into an Excel (Microsoft Corp., Redmond, Washington, USA) table. Raters will resolve disagreements by consensus, and a third rater will arbitrate when consensus is unreachable. The table will include first author, year of publication, country of data collection, proportion female, setting, length of follow-up, type and measure of social support, type and measure of cognitive function, and quantitative results (eg, mean or median scores on instruments measuring functional social support or cognitive function, number or proportion of participants whose scores exceeded some cut-off value on an instrument in question, ORs, relative risks).

\section{Data management}

We will use Covidence (Veritas Health Innovation, Melbourne, Australia) to manage both levels of screening and to record the reasons for excluding citations at either level.

\section{Data synthesis}

Based on previous research looking at the association between functional social support and cognitive function, ${ }^{27}$ we will narratively synthesise the extracted data in groupings based on functional social support subtypes, gender, setting (ie, community dwelling vs institutionalised populations), study design (eg, longitudinal vs cross-sectional studies) and risk of bias level (low, unclear, high).

We will conduct a meta-analysis if we find a pool of included articles with similar characteristics based on the information in the data extraction table (eg, sample age and sex, functional social support measures, cognitive function domains, lengths of follow-up, etc). We will undertake a series of stratified meta-analyses based on the groupings described in the previous paragraph, if numbers permit.

We anticipate that most included articles will measure cognitive function with scores on neuropsychological tests. Therefore, we will convert between-group differences in endpoint test scores into standard mean differences (Hedges's g) and employ the inverse variance method, DerSimonian-Laird estimator for $\pi^{2}$, and a random effects model to conduct any meta-analyses. In some instances, included articles might measure outcomes dichotomously as clinical diagnoses (eg, mild neurocognitive disorder-yes/no). If so, then we will transform the log ORs into standard mean differences using Borenstein et al s formulas. ${ }^{36}$ Statistical heterogeneity will be tested with the $\chi^{2}$ test and $\mathrm{I}^{2}$ statistic, with $\mathrm{p}>0.10$ and $\mathrm{I}^{2}>50 \%$ indicative of high levels of heterogeneity. In the event of high levels of statistical heterogeneity, we will conduct a random-effect meta-regression and examine characteristics such as study design, publication year, setting (eg, community dwelling, long-term care facilities) and sample characteristics (ie, mean age, proportion female).

We will use forest plots, Egger's test and Duval and Tweedie's trim-and-fill method to assess the presence of publication bias if 10 or more articles are included in a meta-analysis. We will use the 'meta' package in R V.3.6.1 (The R Foundation for Statistical Computing, Vienna, Austria) to implement all meta-analytical procedures.

\section{Risk of bias}

Two raters will independently assess the risk of bias of all included articles using the Cochrane Risk of Bias 
Tool-Version 2 for randomised controlled trials ${ }^{37}$ or the Newcastle-Ottawa Scale for observational studies (http:// www.ohri.ca/programs/clinical_epidemiology/oxford. asp). ${ }^{38}$ Raters will resolve disagreements by consensus. A third rater will arbitrate cases for which consensus is unreachable. The proposed systematic review and metaanalysis will be reported in conformity with the Metaanalysis of Observational Studies in Epidemiology ${ }^{39}$ and PRISMA $^{31}$ guidelines. We will also critically appraise the review using the second version of A MeaSurement Tool to Assess systematic Reviews $2 .^{40}$

\section{Timeline for systematic review}

Pilot screening was initiated on 3 December 2019. We anticipate data extraction will begin in April 2020 and a draft manuscript will be completed by December 2020 .

\section{Patient and public involvement}

Patients and the public were not involved in the design or planning of the study.

\section{Ethics and dissemination}

We do not require formal ethics approval because the review will draw on publicly available scientific literature. We will present our findings in a peer-reviewed journal and at relevant conferences. Any amendments made to the protocol during the conduct of the review will be reported in the manuscript.

\section{Author affiliations}

${ }^{1}$ School of Public Health and Health Systems, University of Waterloo, Waterloo, Ontario, Canada

${ }^{2}$ School of Pharmacy, University of Waterloo, Waterlo, Ontario, Canada

${ }^{3}$ School of Planning, University of Waterloo, Waterloo, Ontario, Canada

${ }^{4}$ Department of Psychology, University of Saskatchewan, Saskatoon, Saskatchewan, Canada

${ }^{5}$ Department of Psychology, University of Calgary, Calgary, Alberta, Canada

Acknowledgements The authors wish to thank Rebecca Hutchinson for helping to develop the literature search strategy.

Contributors All authors were involved in the conception and design of the work. ECR and MO drafted the manuscript and the remaining authors critically revised the manuscript for important intellectual content. All authors gave final approval for the manuscript to be published and they agree to be accountable for all aspects of the work. MO is guarantor of the manuscript.

Funding This work was supported by Velux Stiftung grant number 1190.

Competing interests None declared.

Patient and public involvement Patients and/or the public were not involved in the design, or conduct, or reporting, or dissemination plans of this research.

Patient consent for publication Not required.

Provenance and peer review Not commissioned; externally peer reviewed.

Open access This is an open access article distributed in accordance with the Creative Commons Attribution Non Commercial (CC BY-NC 4.0) license, which permits others to distribute, remix, adapt, build upon this work non-commercially, and license their derivative works on different terms, provided the original work is properly cited, appropriate credit is given, any changes made indicated, and the use is non-commercial. See: http://creativecommons.org/licenses/by-nc/4.0/.

\section{ORCID iDs}

Colleen J Maxwell http://orcid.org/0000-0002-7988-2899

Mark Oremus http://orcid.org/0000-0001-8190-253X

\section{REFERENCES}

1 Kim S, Park JL, Hwang HS, et al. Correlation between frailty and cognitive function in non-demented community dwelling older Koreans. Korean J Fam Med 2014;35:309-20.

2 Moritz DJ, KasI SV, Berkman LF. Cognitive functioning and the incidence of limitations in activities of daily living in an elderly community sample. Am J Epidemiol 1995;141:41-9.

3 Kuiper JS, Zuidersma M, Zuidema SU, et al. Social relationships and cognitive decline: a systematic review and meta-analysis of longitudinal cohort studies. Int $J$ Epidemiol 2016;45:dyw089:1169-206.

4 Kuiper JS, Zuidersma M, Oude Voshaar RC, et al. Social relationships and risk of dementia: a systematic review and meta-analysis of longitudinal cohort studies. Ageing Res Rev 2015;22:39-57.

5 Kelly ME, Duff H, Kelly S, et al. The impact of social activities, social networks, social support and social relationships on the cognitive functioning of healthy older adults: a systematic review. Syst Rev 2017;6:259.

6 Evans IEM, Martyr A, Collins R, et al. Social isolation and cognitive function in later life: a systematic review and meta-analysis. $J$ Alzheimers Dis 2019;70:S119-44.

7 Lara E, Martín-María N, De la Torre-Luque A, et al. Does loneliness contribute to mild cognitive impairment and dementia? A systematic review and meta-analysis of longitudinal studies. Ageing Res Rev 2019;52:7-16.

8 Sherbourne CD, Stewart AL. The mos social support survey. Soc Sci Med 1991;32:705-14.

9 Uchino BN, Bowen K, Carlisle M, et al. Psychological pathways linking social support to health outcomes: a visit with the "ghosts" of research past, present, and future. Soc Sci Med 2012;74:949-57.

10 Shankar A, Hamer M, McMunn A, et al. Social isolation and loneliness: relationships with cognitive function during 4 years of follow-up in the English longitudinal study of ageing. Psychosom Med 2013;75:161-70.

11 Zunzunegui M-V, Alvarado BE, Del Ser T, et al. Social networks, social integration, and social engagement determine cognitive decline in community-dwelling Spanish older adults. J Gerontol $B$ Psychol Sci Soc Sci 2003;58:S93-100.

12 Brenowitz WD, Kukull WA, Beresford SAA, et al. Social relationships and risk of incident mild cognitive impairment in U.S. Alzheimer's disease centers. Alzheimer Dis Assoc Disord 2014;28:253-60.

13 Sharifian N, Manly JJ, Brickman AM, et al. Social network characteristics and cognitive functioning in ethnically diverse older adults: the role of network size and composition. Neuropsychology 2019;33:956-63.

14 Schwartz E, Khalaila R, Litwin H. Contact frequency and cognitive health among older adults in Israel. Aging Ment Health 2019;23:1008-16.

15 Giles LC, Anstey KJ, Walker RB, et al. Social networks and memory over 15 years of followup in a cohort of older Australians: results from the Australian longitudinal study of ageing. J Aging Res 2012;2012:856048:1-7.

16 Bennett DA, Schneider JA, Tang Y, et al. The effect of social networks on the relation between Alzheimer's disease pathology and level of cognitive function in old people: a longitudinal cohort study. Lancet Neurol 2006:5:406-12.

17 Sörman DE, Rönnlund M, Sundström A, et al. Social network size and cognitive functioning in middle-aged adults: cross-sectional and longitudinal associations. J Adult Dev 2017;24:77-88.

18 Fratiglioni L, Wang HX, Ericsson K, et al. Influence of social network on occurrence of dementia: a community-based longitudinal study. Lancet 2000;355:1315-9.

19 Sommerlad A, Sabia S, Singh-Manoux A, et al. Association of social contact with dementia and cognition: 28-year follow-up of the Whitehall II cohort study. PLoS Med 2019;16:e1002862.

20 Liao J, Scholes S. Association of social support and cognitive aging modified by sex and relationship type: a prospective investigation in the English longitudinal study of ageing. Am J Epidemiol 2017;186:787-95.

21 Kats D, Patel MD, Palta P, et al. Social support and cognition in a community-based cohort: the Atherosclerosis risk in communities (ARIC) study. Age Ageing 2016;45:475-80.

22 Khondoker M, Rafnsson SB, Morris S, et al. Positive and negative experiences of social support and risk of dementia in later life: an investigation using the English longitudinal study of ageing. $J$ Alzheimers Dis 2017;58:99-108.

23 Oremus M, Tyas SL, Maxwell CJ, et al. Social support availability is positively associated with memory in persons aged $45-85$ years: a cross-sectional analysis of the Canadian longitudinal study on aging. Arch Geronto/Geriatr 2020;86:103962. 
24 Oremus M, Konnert C, Law J, et al. Social support and cognitive function in middle- and older-aged adults: descriptive analysis of CLSA tracking data. Eur J Public Health 2019;29:1084-9.

25 Ge S, Wu B, Bailey DE, et al. Social support, social strain, and cognitive function among community-dwelling U.S. Chinese older adults. J Gerontol A Biol Sci Med Sci 2017;72:S16-21.

26 Pillemer SC, Holtzer R. The differential relationships of dimensions of perceived social support with cognitive function among older adults. Aging Ment Health 2016;20:727-35.

27 Pillemer S, Ayers E, Holtzer R. Gender-stratified analyses revea longitudinal associations between social support and cognitive decline in older men. Aging Ment Health 2019;23:1326-32.

28 Murata C, Saito T, Saito M, et al. The association between social support and incident dementia: a 10-year follow-up study in Japan. Int J Environ Res Public Health 2019;16:239.

29 Rimmer A. Bma meeting: early dementia diagnosis is pointless without support services. BMJ 2016;353:i3505.

30 Moher D, Liberati A, Tetzlaff J, et al. Preferred reporting items for systematic reviews and meta-analyses: the PRISMA statement. PLoS Med 2009;6:e1000097.

31 Liberati A, Altman DG, Tetzlaff J, et al. The PRISMA statement for reporting systematic reviews and meta-analyses of studies that evaluate healthcare interventions: explanation and elaboration. BMJ 2009;339:b2700.

32 Shamseer L, Moher D, Clarke M, et al. Preferred reporting items for systematic review and meta-analysis protocols (PRISMA-P) 2015: elaboration and explanation. BMJ 2015;349:g7647.
33 Rey A. L'examencliniqueenpsychologie. Paris: Presses Universitaires de France, 1964.

34 Survey of Health, Ageing and Retirement in Europe (SHARE). Question CF009_VerbFlulntro. Available: https://www.share-datadocutool.org/ question_constructs/view/2415/lang:1 [Accessed 9 Jan 2020].

35 Corporation R. Rand medical outcomes study: social support survey. Available: https://www.rand.org/health-care/surveys_tools/mos/ social-support.html [Accessed 9 Jan 2020].

36 Borenstein M, Hedges LV, Higgins JPT, et al. Introduction to metaanalysis. West Sussex, UK: John Wiley \& Sons, Ltd, 2009.

37 Sterne JAC, Savović J, Page MJ, et al. Rob 2: a revised tool for assessing risk of bias in randomised trials. BMJ 2019;366:14898:14898.

38 Wells GA, Shea B, O'Connell D, et al. The Newcastle-Ottawa scale (NOS) for assessing the quality of nonrandomised studies in metaanalysesThe.Ottawa Hospital research Institute.. Available: http:// www.ohri.ca/programs/clinical_epidemiology/oxford.asp [Accessed 9 Jan 2020].

39 Stroup DF, Berlin JA, Morton SC, et al. Meta-Analysis of observational studies in epidemiology: a proposal for reporting. JAMA 2000;283:2008-12.

40 Shea BJ, Reeves BC, Wells G, et al. AMSTAR 2: a critical appraisal tool for systematic reviews that include randomised or nonrandomised studies of healthcare interventions, or both. BMJ 2017;358:j4008. 